\title{
Frontières
}

\section{Euthanasie et intégrité}

\section{Enjeux de la loi belge et relation médecin-patient : une réflexion éthique}

\section{Marie-Luce Delfosse}

Volume 24, numéro 1-2, automne 2011, printemps 2012

L’aide médicale à mourir

URI : https://id.erudit.org/iderudit/1013093ar

DOI : https://doi.org/10.7202/1013093ar

Aller au sommaire du numéro

\section{Éditeur(s)}

Université du Québec à Montréal

ISSN

1916-0976 (numérique)

Découvrir la revue

Citer cet article

Delfosse, M.-L. (2011). Euthanasie et intégrité : enjeux de la loi belge et relation médecin-patient : une réflexion éthique. Frontières, 24(1-2), 105-112.

https://doi.org/10.7202/1013093ar

\section{Résumé de l'article}

En 2002, la Belgique a adopté trois lois relatives respectivement à l'euthanasie, aux soins palliatifs et aux droits du patient. Ces lois étendent le champ de l'autonomie décisionnelle du patient. Cependant, s'agissant de l'euthanasie, ne convient-il pas de discerner, au-delà de l'autonomie, un enjeu éthique plus fondamental : l'intégrité morale non seulement du patient mais aussi du médecin ? Tel est le fil conducteur de la réflexion menée ici. Celle-ci s’appuie principalement sur les analyses de l'identité et de l'éthique de Paul Ricoeur dans Soi-même comme un autre, et sur l'approche de la relation médecin-patient d'Henri Ey dans Naissance de la médecine. Ces références conjuguées permettent de proposer des repères éthiques en lien avec l'intégrité morale aux médecins confrontés à une demande d'euthanasie.
Ce document est protégé par la loi sur le droit d'auteur. L'utilisation des services d'Érudit (y compris la reproduction) est assujettie à sa politique d'utilisation que vous pouvez consulter en ligne.

https://apropos.erudit.org/fr/usagers/politique-dutilisation/ 


\section{Résumé}

En 2002, la Belgique a adopté trois lois relatives respectivement à l'euthanasie, aux soins palliatifs et aux droits du patient. Ces lois étendent le champ de I'autonomie décisionnelle du patient. Cependant, s'agissant de l'euthanasie, ne convient-il pas de discerner, au-delà de l'autonomie, un enjeu éthique plus fondamental: l'intégrité morale non seulement du patient mais aussi du médecin? Tel est le fil conducteur de la réflexion menée ici. Celle-ci s'appuie principalement sur les analyses de l'identité et de l'éthique de Paul Ricoeur dans Soi-même comme un autre, et sur l'approche de la relation médecin-patient d'Henri Ey dans Naissance de la médecine. Ces références conjuguées permettent de proposer des repères éthiques en lien avec l'intégrité morale aux médecins confrontés à une demande d'euthanasie.

Mots clés: euthanasie - autonomie intégrité - identité - éthique - autrui.

\begin{abstract}
In 2002, Belgium has enacted three laws respectively on euthanasia, palliative care and patients' rights. These laws widen the range of patient decisional autonomy. Nevertheless, in the case of euthanasia, a more basic ethical consideration is at stake: not only patient's moral integrity but also doctor's moral integrity. This is the main thread of the present article. The analysis is based on Paul Ricoeur's conceptions of identity and ethics in Soimême comme un autre, and on Henry Ey's conception of doctor/patient relationship in Naissance de la médecine. These combined references allow to define ethical indicators supporting moral integrity of doctors confronted with euthanasia requests.
\end{abstract}

Keywords: euthanasia - autonomy integrity - identity - ethics - fellow being.

\section{EUTHANASIE ET INTÉGRITÉ}

\section{Enjeux de la loi belge et relation médecin-patient: une réflexion éthique}

\author{
Marie-Luce Delfosse, Ph. D., \\ professeure d'éthique et de bioéthique, \\ Facultés universitaires Notre-Dame de la Paix, \\ Namur (Belgique).
}

La Belgique a adopté le 28 mai 2002 une loi relative à l'euthanasie qui dépénalise celle-ci sous certaines conditions ${ }^{1}$; le 14 juin de la même année, elle a adopté une loi relative aux soins palliatifs, et le 22 août une loi relative aux droits du patient. L'adoption quasi simultanée de ces trois lois souligne bien leur interaction (Van Neste, 2012, section "Autres lois complémentaires»). Les deux premières visent à organiser des possibilités en fin de vie, et la troisième s'attache à définir les principes qui doivent régir les relations de soins entre le médecin et son patient. L'objectif commun de ces lois est de donner juridiquement consistance à l'autonomie décisionnelle du patient qui y occupe une place centrale. Dans la loi relative aux droits du patient et dans la loi relative à l'euthanasie, cette autonomie est affirmée d'emblée (loi droits du patient, art. 5 ; loi euthanasie, art. 2); dans la loi relative aux soins palliatifs, elle constitue à la fois un des objectifs poursuivis par de tels soins et la condition des examens et traitements (loi soins palliatifs, art. 2 et 7).

Quels sont les enjeux philosophiques de la loi relative à l'euthanasie? Quels repères éthiques peut-on en dégager pour les médecins? Telles sont les questions que j'envisagerai dans cet article.

\section{LA LOI BELGE RELATIVE À L'EUTHANASIE: QUEL ENJEU ÉTHIQUE ?}

\section{ÉTENDRE L'AUTONOMIE DU PATIENT?}

Les débats qui ont précédé l'adoption de la loi relative à l'euthanasie ont été fort importants à la fois par leur ampleur, leur durée et la retransmission télévisée intégrale et sur le vif qui en fut faite. Ceux qui soutenaient une initiative législative en cette matière ont fortement mis en évidence l'autonomie décisionnelle des personnes. En effet, leur volonté déclarée était d'étendre le champ de cette autonomie à un type de décision qui, jusqu'alors, 
y échappait. Les témoignages, livrés par ailleurs, de Roger Lallemand, sénateur socialiste, et de Pierre de Locht, théologien catholique, l'un et l'autre figures intellectuelles marquantes de la vie publique en Belgique, sont révélateurs à cet égard ${ }^{2}$. Selon le premier:

La réforme législative veut fonder une éthique de la responsabilité, celle qui reconnaît à chaque personne adulte le droit d'apprécier la valeur de sa vie et qui, dans des souffrances inapaisables, provoquées par une maladie aussi grave qu'incurable, avec la solidarité d'un médecin, fonde la possibilité d'y mettre fin. En vérité, il s'agit de réaffirmer, face à la vie et à la mort, une autonomie de la personne, une "souveraineté » sur elle-même (Lallemand et de Locht, 2001, p. 8). Pour le second:

Je suis vivant, avec le privilège en même temps que la charge redoutable d'exister en étant conscient de l'être. Dès lors, avec le désir de faire de mon existence quelque chose d'heureux et de cohérent pour moi comme pour les autres. Cette cohérence foncière d'un être avec lui-même, qui me paraît essentielle et vitale pour moi, en tant que personne humaine, je la souhaite également pour les autres. J'attends donc que la société s'organise afin de favoriser autant que possible ce que chacun veut faire de sa vie, dans le respect d'une même aptitude chez les autres (Lallemand et de Locht, 2001, p. 9).

\section{QUELLE CONCEPTION DE L'AUTONOMIE} DANS LA LOI?

La loi insiste à la fois sur le caractère libre de la demande du patient et sur le fait que l'euthanasie ne peut être réalisée qu'à sa demande. Cependant, cette autonomie, doublement affirmée, n'est pas celle d'un sujet isolé. Bien au contraire, elle est inscrite d'emblée dans l'intersubjectivité. L'article 3 de la loi pose en effet que le médecin «doit arriver, avec le patient, à la conviction qu'il n'y a aucune autre solution raisonnable dans sa situation et que la demande du patient est entièrement volontaire ». Il doit également s'assurer de la volonté «réitérée» du patient, et pour ce faire il doit avoir avec celui-ci «plusieurs entretiens, espacés d'un délai raisonnable au regard de l'évolution de l'état du patient». La loi pose ainsi des conditions qui vont dans le sens d'une information du patient sur son état de santé et les possibilités thérapeutiques et palliatives envisageables, ainsi que d'une écoute attentive et d'un dialogue nourri avec lui. Elle requiert en outre que le médecin s'entretienne avec l'équipe soignante en contact régulier avec le patient, et qu'il consulte un, voire deux médecins indépendants. Se dégage ainsi de la loi une conception du patient comme sujet autonome en même temps que relié à autrui et construisant avec lui sa décision qui doit être actée par écrit - dans un réel partenariat qui engage leur responsabilité mutuelle ${ }^{3}$.

LA PRISE AU SÉRIEUX

\section{DE L'AUTONOMIE DES PATIENTS}

DE MÊME QUE LA VOLONTÉ

DE METTRE FIN À L'INSÉCURITÉ

JURIDIQUE À LAQUELLE

ÉTAIENT CONFRONTÉS

LES MÉDECINS ONT ÉTÉ

DES MOTIFS DÉTERMINANTS

EN FAVEUR DE L'ADOPTION

D'UNE LÉGISLATION

QUI RESPECTE LES PARTIES

EN PRÉSENCE ET DÉFINISSE LEURS

RESPONSABILITÉS MUTUELLES.

Cette conception permet de récuser toute approche de l'autonomie qui aboutirait à instrumentaliser un des termes de la relation. Le patient peut demander l'euthanasie, mais il ne peut l'exiger du médecin qui peut d'ailleurs refuser de la pratiquer, à condition d'en informer le patient «en temps utile» et d'en préciser les raisons (loi euthanasie, art. 14). Le droit de demander l'euthanasie, reconnu aujourd'hui au patient en Belgique, est donc bien distinct d'un droit à l'euthanasie comme le souligne Fernand Van Neste dans ce numéro (Van Neste, 2012, section «Demande volontaire, réfléchie, répétée et sans pression extérieure», in fine). Quant au médecin, redisons-le, il ne peut pratiquer une euthanasie qu'à la demande du patient. Comme le soulignait bien Roger Lallemand, le régime juridique antérieur à la loi du 28 mai 2002 mettait en péril le dialogue entre le malade et son médecin car il développait le secret des pratiques (Lallemand et de Locht, 2001: 29). Dans tous les pays du monde, des euthanasies ont été pratiquées, souvent au nom de l'état de nécessité, sans que le patient ait eu voix au chapitre. La prise au sérieux de l'autonomie des patients de même que la volonté de mettre fin à l'insécurité juridique à laquelle étaient confrontés les médecins ont été des motifs déterminants en faveur de l'adoption d'une législation qui respecte les parties en présence et définisse leurs responsabilités mutuelles.

\section{UN AUTRE ENJEU ÉTHIQUE ?}

\section{THÈSES}

Si les déclarations de Roger Lallemand et de Pierre de Locht convergent dans l'affirmation de l'autonomie du patient, la seconde suggère cependant une question : cette autonomie est-elle en définitive l'enjeu éthique final du droit de demander l'euthanasie? Pierre de Locht évoque «la cohérence d'un être avec lui-même » qu'il lie à l'autonomie de décision de chacun. L'interrogation rebondit alors car c'est l'enjeu même de la demande d'euthanasie formulée par le patient reconnu autonome qui se trouve cette fois au centre de l'attention. En mettant en avant la cohérence, Pierre de Locht laisse entendre que la décision du patient appelle une délibération intérieure, voire une justification par rapport à soi-même, qui peut, le cas échéant, être entendue par les proches et la société, et peut engager le rapport à l'instance que l'on considère comme donatrice de la vie. Les deux thèses que je voudrais soutenir dans cet article sont les suivantes. Reconnaître l'autonomie du patient jusqu'à lui conférer le droit de demander l'euthanasie renvoie à un enjeu éthique plus fondamental que l'autonomie, tout en étant indissociable de celleci: le respect de l'intégrité de ce patient. Cependant, dans ces situations, ce n'est pas l'intégrité du seul patient qui est en jeu, c'est aussi celle du médecin, et le respect de leur intégrité respective est décisif pour assurer la qualité éthique de l'euthanasie.

\section{AU FIL D'UN CHEMINEMENT}

INTERDISCIPLINAIRE

Ces deux thèses se sont imposées à moi à la faveur d'un parcours à la fois personnel et partagé. Je me permets de l'évoquer car il me semble révélateur de la dynamique propre de l'éthique appliquée. Le comité d'éthique hospitalière dont je suis membre avait été interpellé sur différentes questions concernant la fin de la vie, notamment les formulaires de non-réanimation. Plutôt que de proposer des réponses ponctuelles, il avait décidé d'entreprendre une réflexion globale sur cette période de la vie. C'est au cours de celle-ci que la notion d'intégrité s'est glissée dans la discussion, de façon subreptice, au départ d'une allusion qui y était faite, brièvement et dans 
un sens fort spécifique dans un texte centré sur l'identité confessionnelle des institutions, ce qui n'était pas alors notre préoccupation. Des membres se sont saisis de cette notion, et l'ont reprise indépendamment du texte (et du contexte) où ils l'avaient rencontrée: ils estimaient qu'elle correspondait à ce qu'ils ressentaient intuitivement dans certaines situations de fin de vie auxquelles ils étaient confrontés dans leur pratique professionnelle. Chacun des membres du comité a progressivement scruté plus avant la notion d'intégrité au regard de ses pratiques, donc pour les uns de façon très concrète, et pour moi philosophiquement. Ce qui mérite d'être souligné dans cette démarche qui prit un temps certain - et je pense que prendre ce temps était indispensable -, c'est l'interaction qui s'est établie entre l'approche réalisée par les praticiens (médecins, infirmiers, agent pastoral, juristes) et la réflexion philosophique. Cette dernière ne fut pas du tout la «norme morale» sur laquelle aligner ensuite les pratiques médicales et soignantes. C'est bien plutôt un appui mutuel et une convergence qui se sont tissés progressivement: l'élucidation philosophique a permis de déployer les intuitions vécues dans les pratiques de soin, et celles-ci ont encadré cette réflexion en amont et en aval: en la stimulant et en permettant d'en mettre à l'épreuve la justesse, l'adéquation «au plus près » des situations. Au terme de ce parcours, c'est en termes de vérité que tous les membres du comité se sont reconnus dans le travail réalisé. Il en fut de même pour l'institution qui a accepté que les conclusions de ce travail deviennent des lignes directrices pour la pratique en son sein.

Le texte qui suit ne reprend pas la présentation de notre réflexion commune, telle que nous l'avons soumise à l'institution; il s'attache plutôt à mettre au jour l'argumentation philosophique qui la soustend et dont la portée peut être significative au-delà du cadre local dont elle est issue.

\section{ÉTAPES DE L'ARGUMENTATION}

Partant de la thèse qu'au-delà de l'autonomie, c'est l'intégrité qui constitue l'enjeu fondamental d'une demande d'euthanasie, je montrerai d'abord la teneur à la fois individuelle et relationnelle de ce concept dans un parcours guidé par Charles Taylor et Paul Ricoeur. L'intégrité apparaîtra ainsi comme un enjeu partagé dans la relation entre le patient et son médecin, que je scruterai en m'appuyant sur Henri Ey. En référence à ces auteurs, je proposerai alors quelques repères destinés à guider l'agir des médecins dans les situations éminemment délicates de demande d'euthanasie, et j'indiquerai en finale quelques-uns des changements que la loi relative à l'euthanasie a apportés dans les pratiques et les mentalités.

\section{APPROCHES DE L'INTÉGRITÉ}

\section{DÉFINITIONS}

À première vue, il peut sembler paradoxal de voir en l'intégrité l'enjeu décisif d'une demande d'euthanasie, alors que celle-ci vise précisément à mettre fin à l'intégrité physique. Une analyse plus attentive lève cependant ce paradoxe. Elle s'avère d'autant plus nécessaire que le concept d'intégrité, souvent invoqué dans les textes internationaux de droit et d'éthique médicale, est rarement explicité. Avant d'examiner son sens face à l'euthanasie, adoptons cette définition générale comme fil conducteur de la réflexion: l'intégrité est «un état inaltéré de complétude que l'on doit respecter» (Hermeren, 2001: 542).

Qu'en résulte-t-il pour la compréhension de l'intégrité physique? Celle-ci ne se résume pas aux normes «objectives» de fonctionnement physique définies par la médecine scientifique. On le sait, de telles normes expriment des moyennes observables. Or, le «normal» qu'elles énoncent est problématique. En effet, dans leur pratique, lors de la mise au point des traitements, les médecins et les soignants sont constamment confrontés à la singularité biologique des patients. Une évidence s'impose alors à eux: l'intégrité physique se décline de manière individuelle et est indissociable des possibilités intrinsèques d'adaptation du patient à son milieu ${ }^{4}$. Elle ne peut donc se comprendre uniquement en référence à une norme extérieure au patient lui-même.

L'intégrité morale renvoie à la capacité des individus de suivre leur conscience, de prendre des résolutions, et de résister aux tentatives de les faire changer d'avis (Hermeren, 2001: 543). Elle n'exprime pas la rigidité de qui se refuse à toute discussion, mais bien plutôt la dynamique morale et existentielle propre à chaque personne qui conduit celle-ci à se forger un regard propre sur le monde et sur soi, regard en fonction duquel elle prendra des décisions. Elle est ainsi une quête personnelle d'autonomie.

L'intégrité morale fait en quelque sorte écho à la norme biologique produite par le vivant singulier, et en laquelle consiste l'intégrité physique de celui-ci: elle aussi renvoie à la singularité de chacun et à la relation spécifique qu'il a nouée avec son milieu. Ensemble, elles constituent une histoire dans laquelle le corps et l'esprit sont étroitement intriqués.

\section{AUTONOMIE ET INTÉGRITÉ}

On a pu voir que des liens étroits unissent l'intégrité morale et l'autonomie. Cette dernière doit cependant être scrutée davantage. En effet, sa signification et sa portée constituent actuellement l'objet de nombreux débats qui - fait remarquable - s'articulent souvent autour des conceptions de Kant et de Mill, présentées comme opposées. Or, si ces deux conceptions sont effectivement bien différentes, il faut cependant reconnaître qu'elles se rejoignent formellement dans l'affirmation de l'intégrité morale des sujets. Poser que la loi morale universelle est constitutive de soi ou considérer que la liberté comprise autodétermination doit être préservée tant qu'elle ne cause pas de tort à autrui convergent en effet dans la reconnaissance que l'être humain est capable de prendre ses décisions par lui-même.

À l'époque moderne, cette affirmation avait une portée militante: l'enjeu était alors de faire valoir, aux plans moral et surtout politique, la dignité intrinsèque de chaque être humain, dont l'autonomie kantienne ou l'autodétermination millienne étaient l'expression. Dans les sociétés contemporaines qui mettent en œuvre une culture démocratique, cette problématique est dépassée, du moins formellement. L'autonomie y est reconnue et fortement affirmée, et elle se décline le plus souvent en autodétermination, Mill l'emportant à cet égard sur Kant dans les mentalités dites post-modernes.

Marcel Gauchet constate combien l'autonomie est devenue une réalité inscrite dans la trame même des sociétés occidentales, qu'elle restructure de fond en comble, tout en perdant cependant "son ressort dynamique» (Gauchet, 1998: 75). Ceci pose, on le sait, de multiples problèmes, souvent soulignés, notamment celui de la consistance du lien social, du repli sur les droits subjectifs, de l'épuisement de la référence à des objectifs politiques communs, de l'oubli du politique comme «vivre ensemble» plus fondamental que la politique (Gauchet, 2002: 275-303).

Reconnaître l'autonomie comme un fait n'est pourtant pas le dernier mot, comme le montre Charles Taylor qui refuse une appréciation unilatéralement négative de l'individualisme contemporain (Taylor, 1994). Même s'il estime que les manifestations contemporaines de cet individualisme posent d'énormes problèmes et sont finalement autodestructrices, il soutient qu'elles procèdent d'un idéal d'authenticité qui est, lui aussi, spécifiquement moderne. En effet, avec la modernité, les individus, posés comme les constituants premiers de la société, 
deviennent en même temps responsables de penser par eux-mêmes. Pour ce faire, ils doivent, selon Taylor, se mettre à l'écoute de leur voix intérieure. Si Kant décline celle-ci en termes d'universalité, d'autres philosophes, tel Herder, l'entendront, eux, comme spécificité personnelle. Dès lors, pour Taylor, l'authenticité exige l'écoute de soi, la fidélité à sa propre originalité et l'incarnation de celle-ci dans des paroles et des actes qui la modéleront à son tour, tout en assumant le risque de s'opposer aux règles morales et sociales ambiantes. S'y ajoutent deux autres conditions auxquelles la quête d'authenticité doit satisfaire pour ne pas se dévoyer: "une ouverture à des horizons de signification (car sans eux la création perd la perspective qui peut la sauver de l'insignifiance) et une définition de soi dans le dialogue» (Taylor, 1994: 74).

La quête d'authenticité ainsi conçue n'est donc nullement solipsiste. Taylor la distingue soigneusement d'une forme de liberté fort valorisée dans la culture contemporaine, et qu'il appelle la « liberté autodéterminée »: se considérer comme libre parce que l'on décide et choisit soimême, en s'affranchissant de toutes les obligations extérieures. Une telle liberté est autoréférentielle: le moi en est la seule matière et la seule référence. Elle se réduit à la liberté de choix, et n'est qu'une expression partielle et dégradée de l'idéal d'authenticité qu'elle menace en définitive de détruire.

Tel que le conçoit Taylor, l'idéal moral d'authenticité se révèle être en définitive un idéal d'intégrité morale, la fidélité à soi se construisant dans l'interaction avec autrui. Comment comprendre plus avant cette double dimension? La réflexion de Paul Ricoeur sur le soi constitue à cet égard un jalon éclairant en même temps qu'elle donne une profondeur nouvelle à notre réflexion sur l'intégrité.

\section{LE SOI, POINT NODAL DE L'INTÉGRITÉ MORALE}

Nœud de la problématique de l'intégrité morale, le soi suscite deux questions. Se constitue-t-il par exclusion de l'autre ou par sa relation avec lui? Quelle est sa teneur intrinsèque? Paul Ricoeur a traité ces deux questions dans l'ouvrage qu'il a intitulé de manière significative Soi-même comme un autre. J'évoquerai ici quelques aspects de sa réflexion qui permettent de progresser dans la nôtre.

\section{SOI ET AUTRUI}

D'emblée, Ricoeur insiste: "soi » et pas «je» (Ricoeur, 1990: 11). Car le soi qu'il scrute n'est pas le Cogito cartésien, il ne se donne pas comme une évidence qui s'impose. Il n'est pas davantage le

NGEUD DE LA PROBLÉMATIQUE DE L'INTÉGRITÉ MORALE,

LE SOI SUSCITE DEUX QUESTIONS. SE CONSTITUE-T-IL

PAR EXCLUSION DE L'AUTRE OU PAR SA RELATION AVEC LUI?

«je pense» kantien, fondement dernier de toutes les propositions empiriques et transcendantales. Pour Ricoeur, il implique si intimement l'altérité qu'il ne peut se penser sans l'autre.

La dialectique entre le soi et l'autre, présente tout au long du livre, se déploie pleinement dans les études sur l'éthique (p. 199-344). On le sait, Ricoeur conçoit cette dernière comme une démarche animée d'une dynamique intrinsèque: trois moments - la visée éthique, la norme morale, la sagesse pratique - s'enchaînent et se déplient chacun en trois composantes étroitement liées les unes aux autres - soi, autrui, les institutions.

Afin de donner consistance à la visée d'une vie bonne, moment initial de la démarche éthique, Ricoeur montre que chacun est invité à une interprétation de soi et de son action. Envisagée sous l'angle de la responsabilité, cette autoréflexion devient estime de soi dans la prise de conscience par le sujet qu'il est un être capable d'initiatives et de choix (p. 212). Cette estime de soi demeure toutefois abstraite tant qu'elle ne s'inscrit pas dans une structure dialogique. Celle-ci met au jour une demande de reconnaissance réciproque, et instaure un schéma d'estime croisée: autrui est estimable comme moimême. Elle révèle dans le soi une spontanéité bienveillante envers autrui, que Ricoeur dénomme «sollicitude» (p. 211), basée fondamentalement sur l'échange entre donner et recevoir (p. 220), et dont le secret, écrit-il, est la réciprocité des insubstituables: les personnes qui constituent les pôles de l'échange sont irremplaçables (p. 225-226). Cependant, «la sollicitude ne s'ajoute pas du dehors à l'estime de soi, mais [...] elle en déplie la dimension dialogale» si bien que «l'estime de soi et la sollicitude ne puissent se vivre et se penser l'une sans l'autre» (p. 212). Toutefois, "l'autre, c'est aussi l'autre que le “tu”» (p. 227). C'est pourquoi cette structure dialogique reste incomplète sans la référence à des institutions justes (p. 202). La visée éthique est ici sens de la justice, antérieur à la construction de systèmes juridiques et à la définition de politiques.

Le lien intrinsèque entre soi et autrui se révèle aussi dans les deux autres moments de la démarche éthique. Je rappelle ici brièvement les deux premières composantes de celle-ci - soi et autrui
-, qui sont décisives pour la suite de la réflexion.

Toutes les composantes de la visée éthique doivent se soumettre à l'épreuve de la norme morale afin de s'épurer des dérives dont elles peuvent être porteuses. Ricoeur insiste à la fois sur la continuité et la discontinuité entre visée éthique et norme morale. La norme morale prolonge la visée éthique et la transpose sur le plan d'exigences caractérisées par leur prétention à l'universalité et un effet de contrainte, tout en n'en constituant qu'une effectuation limitée (p. 201).

Ricoeur met clairement en évidence cette continuité et cette discontinuité. Pour lui, «le respect de soi est l'aspect que revêt l'estime de soi sous le régime de la norme» (p. 201). Pensé dans la ligne de Kant, ce respect est celui que se doit l'être humain en tant qu'il est capable d'autolégislation morale, d'autonomie. Il se prolonge en respect de l'humanité dans sa propre personne comme dans celle de toute autre, ainsi que l'exprime la célèbre formule de Kant: "Agis de telle sorte que tu traites l'humanité aussi bien dans ta personne que dans celle de toute autre, toujours en même temps comme une fin, jamais simplement comme un moyen" (cité p. 258, note 1). Toutefois, Ricoeur souligne la tension interne de cette formule. Comme la sollicitude, elle est dialogique et vise à établir la réciprocité entre les personnes. Mais en envisageant celles-ci sous l'idée universelle de l'humanité, elle se limite à l'affirmation de leur pluralité. Elle reste ainsi indifférente à l'altérité qui est pourtant à la racine même de la pluralité et dont l'intuition est inhérente à la sollicitude (p. 261).

L'application des normes aux situations concrètes peut cependant susciter des conflits de devoirs. Ainsi en est-il quand l'altérité des personnes ne peut être coordonnée avec l'universalité de l'humanité: «le respect tend alors à se scinder en respect de la loi et respect des personnes» (Ricoeur: 305). Dans ces situations, les normes renvoient, au-delà d'elles-mêmes, vers une conviction plus décisive qu'elles, celle qui anime la sagesse pratique. Cette dernière met en œuvre un jugement moral attentif à la particularité des situations et peut conduire à donner la priorité au respect des personnes au nom d'une sollicitude soucieuse de la singularité des personnes. Cette conviction n'est pas 
arbitraire : elle fait appel aux ressources du sens éthique originaire, celles de la visée, que la reprise opérée par la norme laisse hors d'elle. Elle ne constitue pourtant pas un retour pur et simple à la sollicitude «naïve» de la visée éthique : celle qui est en œuvre ici est «critique», elle a traversé l'épreuve de la confrontation à la norme du respect et des conflits suscités par celui-ci (p. 318). C'est précisément cette traversée qui distingue la sagesse pratique d'une éthique de situation.

LA TENEUR DU SOI

À CE NIVEAU DE RELATION, LE MÉDECIN CESSE D'ÊTRE SEULEMENT MÉDECIN ET EST AUSSI L'HUMAIN, À LA FOIS SEMBLABLE À SON MALADE QUI VIT L'EXPÉRIENCE DE LA MALADIE SUR LE REGISTRE DU DÉSIR ET DE L'ANGOISSE, ET EN MÊME TEMPS DIFFÉRENT DE LUI ET DEVANT LE RESTER. INTERPELLÉ PAR LA PLAINTE DU MALADE, IL DOIT Y RÉPONDRE NON SEULEMENT TECHNIQUEMENT MAIS AUSSI MORALEMENT. LA FONCTION MÉDICALE EST DONC AMBIGUË : SCIENTIFIQUE ET TECHNIQUE À L'ÉGARD DE LA MALADIE, MORALE À L'ÉGARD DU MALADE.

Outre cette dynamique intrinsèque de l'éthique qui révèle le lien indissociable entre le soi et l'autre, Ricoeur s'interroge sur la teneur même du soi: comment comprendre son identité constitutive? Il montre que l'identité, confrontée à la question de la permanence dans le temps, peut se comprendre de deux manières: comme le semblable, le même; comme le maintien de soi. La première, dénommée «identité-idem» ou mêmeté, renvoie à un invariant relationnel, à une structure permanente dans le temps; Ricoeur en a vu longtemps l'emblème dans le caractère. La seconde, dénommée «identité-ipse» ou «ipséité », renvoie - on le saisit d'emblée - à une autre forme de permanence de temps; son emblème est la fidélité à la parole donnée.

Ricoeur revient sur sa conception antérieure et reprend l'analyse du caractère: il montre qu'il faut se garder d'interpréter celui-ci de façon statique et solipsiste et que les deux types d'identité peuvent s'y conjuguer. "Ensemble des dispositions durables à quoi on reconnaît une personne» (p. 146), le caractère a une histoire dans laquelle la place d'autrui est décisive. En effet, la disposition renvoie à l'habitude avec sa double dimension: être déjà acquise et être en voie d'acquisition. Elle renvoie aussi aux identifications acquises: identification aux valeurs, idéaux et modèles dans lesquels la personne se reconnaît et par lesquelles «de l'autre entre dans la composition du même» (p. 146). En même temps, «un élément de loyauté [...] s'incorpore ainsi au caractère et le fait virer à la fidélité, donc au maintien de soi» (p. 147). Si l'idem et l'ipse se conjuguent de la sorte dans le caractère, l'ipse s'y voit cependant recouvert presque complètement par l'idem. Par contre, la parole tenue dans la fidélité à

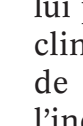

\section{de} l'individu concret qui se trouve face à lui. Autrement dit, «le regard du médecin [...] peut saisir la souffrance, l'épreuve et la menace qui constituent [pour le patient] sa maladie comme une manifestation de la vulnérabilité de son corps [...] corruptible et transitoire» $(\mathrm{Ey}: 11)$.

En même temps que s'opère ce travail de compréhension de la maladie, un espace de réciprocité relationnelle essentiellement humaine ou morale s'organise aussi entre le médecin et le malade: «le médecin, dans sa rencontre, dans son dialogue ou dans sa communication avec le malade, ne perçoit jamais la maladie sans percevoir le sujet qui, en étant affecté, la lui montre ou la lui cache» (Ey: 12). Il perçoit donc le malade comme quelqu'un qui s'adresse à lui et lui demande son aide, "un alter ego qui vient à sa rencontre avec les forces et les modalités de sa propre subjectivité» (p. 11). Or pour le médecin comme pour le malade, cet espace est hanté par les fantasmes qu'éveille l'expérience vécue du mal. Dès lors, pour le médecin, «l'objectivité de la maladie [...] est pour ainsi dire immergée dans les rapports d'intersubjectivité rebelles à toute codification exacte et rationnelle» (p. 12).

À ce niveau de relation, le médecin cesse d'être seulement médecin et est aussi l'humain, à la fois semblable à son malade qui vit l'expérience de la maladie sur le registre du désir et de l'angoisse, et en même temps différent de lui et devant le rester. Interpellé par la plainte du malade, il doit y répondre non seulement techniquement mais aussi moralement. La fonction médicale est donc ambiguë: scientifique et technique à l'égard de la maladie, morale à l'égard du malade. Comme le dit bien Ey, «le médecin tient au malade un discours à deux voix» (p. 12). Comment peut-il harmoniser celles-ci, particulièrement lorsqu'il est en relation avec un patient qui demande l'euthanasie? 


\section{REPÈRES}

POUR TOUTE RELATION

MÉDECIN-PATIENT

Dans la foulée d'Henri Ey, il importe d'abord de se rappeler qu'il n'est pas de médecine en dehors de la relation - à la fois scientifique, technique et morale entre un médecin et son patient qui lui demande de partager, voire d'assumer son tourment (p. 5).

Ensuite, comme le souligne aussi Ey, il faut se rappeler que le mystère de la douleur, qui est au cœur de la relation médecin-patient, affecte le patient dans sa globalité. En effet, même si la maladie est localisée dans un point particulier du corps, elle est ressentie par le patient comme un mal, une menace de décomposition, un blocage qui affecte le cours de son existence. La douleur atteint donc le corps comme l'esprit, et témoigne de leur étroite union, au-delà de tout dualisme.

\section{FACE À UNE DEMANDE D'EUTHANASIE}

Si le patient peut réagir à sa maladie par une demande de restauration de sa santé, il peut aussi considérer que cette altération le dénature si radicalement qu'elle constitue une atteinte à son intégrité physique et morale. Il peut alors vouloir que les moyens de surmonter ou de pallier cette perte d'intégrité ne soient pas mis en œuvre, et voir en sa maladie la préfiguration de sa mort. Une telle appréciation est subjective. Même si elle est à confronter aux alternatives possibles, le patient reste en définitive le seul juge car l'expérience de la douleur est, elle aussi, subjective. C'est ce qu'évoque subtilement Noëlle Châtelet dans La dernière leçon qui raconte sa familiarisation progressive avec la décision de sa mère de mettre fin à ses jours:

«Maman, es-tu sûre que c'est le moment?

- Oui, ma chérie, je suis sûre. Je le sais. Je le sens. Moi seule peux le sentir, le savoir.»

Toi seule? Évidemment toi seule. Qui d'autre que toi pouvait en effet les apprécier, dans leur réalité, les fameuses limites de l'insupportable [...] ? Qui d'autre pouvait s'arroger le droit de contester ou de s'opposer à l'intime conviction de ta dignité ou de ton indignité? Tes enfants? Au nom de l'amour? Non, non, même pas eux! (Châtelet, 2005, p. 52-53).

Le médecin doit-il pour autant s'incliner devant la décision d'un patient qu'il estime injustifiée? L'approche de Ey converge ici avec la réflexion éthique que propose Ricoeur. Ey montre que le médecin doit adopter une attitude secourable à l'égard de son patient: celle-ci n'est possible que grâce à la proximité qui s'instaure entre lui et son patient, qui, tout à la fois, sont semblables et doivent rester différents, et à cet égard sont insubstituables. Ricoeur appelle cette attitude secourable «sollicitude». Il en voit l'expression dans la Règle d'or: «ne fais pas à autrui ce que tu ne voudrais pas qu'il te fasse», "ce que tu voudrais qu'autrui fasse pour toi, fais-le pour lui ». Cette formulation montre clairement la dissymétrie entre les partenaires. Or cette dissymétrie ouvre la possibilité de la violence, c'est-à-dire du pouvoir exercé par une volonté sur une autre. C'est pourquoi la sollicitude spontanée doit s'épurer en se confrontant à la norme du respect des personnes. La norme ne pourra cependant donner le dernier mot car elle place le médecin devant un conflit de devoirs: respecter la vie et par là même l'engagement qu'il a pris en prêtant serment ou respecter la personne dans cette volonté extrême. Le respect, dans cette situation tragique, s'avère impuissant à dicter la conduite. Il doit renvoyer à la sollicitude, non pas celle «naïve» de la visée éthique, mais celle «critique » qui s'est confrontée aux normes morales et aux conflits que celles-ci suscitent. Réfléchissant à la promesse et à la norme morale qu'elle implique - la fidélité aux engagements pris - Ricoeur remarque: "L'obligation de se maintenir soi-même en tenant ses promesses est menacée de se figer dans la raideur stoïcienne de la simple constance, si elle n'est pas irriguée par le vœu de répondre à une attente, voire une requête venue d'autrui » (Ricoeur, 1990 : 311). L'identitéipse, évoquée plus haut, est ici mise en question. La réponse de Ricoeur, inspirée de Gabriel Marcel, est la disponibilité: «C'est à l'autre que je veux être fidèle » (p. 311). Et il précise:
La disponibilité est cet exode qui ouvre le maintien de soi sur la structure dialogique instituée par la Règle d'or. Celle-ci, en tant que règle de réciprocité posée dans une situation initiale dissymétrique, établit l'autre dans la position d'un obligataire qui compte sur moi et fait du maintien de soi une réponse à cette attente $[\ldots]:$ : tu peux compter sur moi ». Ce compter sur relie le maintien de soi, dans sa teneur morale, au principe de réciprocité fondé sur la sollicitude (p. 311-312).

On l'a vu, cette disponibilité confronte le médecin à une question difficile: comment être à la fois disponible pour autrui et donc dépossédé de soi, tout en étant fidèle aux valeurs morales qu'il s'est engagé à servir ? Pour Ricoeur, la réponse est dans la sagesse pratique qui consiste à inventer les conduites qui satisferont le plus à l'exception que demande la sollicitude en trahissant le moins possible l'impératif du respect de l'humanité dans sa personne comme dans celle de toute autre (p. 312). Qu'entendre par là? Non pas un jeu avec les limites. Mais au contraire, l'invention d'un comportement inédit qui respecte l'humanité du patient tout en étant attentif à la particularité de sa situation.

\section{L'INTÉGRITÉ DU MÉDECIN}

\section{FACE À L'INTÉGRITÉ DU MALADE}

Comment cette indication générale peut-elle se concrétiser et se préciser pour le médecin quand son patient lui demande, au nom de sa cohérence existentielle, de mettre fin à sa vie? Les réflexions de Ricoeur et de Ey permettent d'exclure plusieurs attitudes. La pitié où, comme le note très justement Ricoeur, «le moi jouit secrètement d'être épargné». L'identification au patient, car, comme l'indique Ey, le médecin doit demeurer un alter ego à l'égard de son patient. La

AU NOM D'UNE CONVICTION FERME ET BIEN RÉFLÉCHIE, LE PATIENT PEUT DIRE : «MON TEMPS EST ACCOMPLI ». DE SON CÔTÉ, LE MÉDECIN DEVRAIT POUVOIR DIRE : «JE SUIS EN ACCORD AVEC MOI-MÊME EN SATISFAISANT À CETTE DEMANDE DU PATIENT, PARCE QUE CELLE-CI EXPRIME NON SEULEMENT SA COHÉRENCE EXISTENTIELLE, MAIS AUSSI LA MIENNE DANS CETTE SITUATION PRÉCISE, AVEC LUI EN PARTICULIER ». 
soumission pure et simple à la volonté d'autrui sous peine de se nier soi-même en devenant un pur exécutant technique des volontés de celui-ci. La démission par rapport à la norme fondamentale de l'éthique médicale et du droit, le respect de la vie.

Quelle attitude positive promouvoir? Comment le médecin peut-il rester fidèle à lui-même et affirmer sa propre intégrité morale tout en recevant la demande d'euthanasie de son patient? Cette affirmation de soi se construit bien évidemment en interaction avec le patient, dans le processus de communication, de dialogue et d'empathie déjà évoqué. Mais cela n'est pas suffisant. Car l'interaction dont il s'agit ici se joue aussi dans l'intériorité du médecin, dans la reprise réflexive qu'il opère de la situation afin d'en cerner le sens et les particularités, de tenter de ressaisir la cohérence existentielle que le patient exprime, de confronter cette dernière à sa propre cohérence existentielle. Or, l'attente que le patient exprime dans une demande d'euthanasie ébranle d'abord le médecin dans ses propres convictions. En effet, elle le met devant la responsabilité de poser des actes contraires à son engagement initial de respecter la vie. Au nom d'une conviction ferme et bien réfléchie, le patient peut dire: "mon temps est accompli». De son côté, le médecin devrait pouvoir dire: "je suis en accord avec moi-même en satisfaisant à cette demande du patient, parce que celle-ci exprime non seulement sa cohérence existentielle, mais aussi la mienne dans cette situation précise, avec lui en particulier». Autrement dit, mise en question par la demande du patient, la sollicitude du médecin à son égard ne se structure plus d'abord en référence à la norme générale du respect d'autrui dans sa vie. Ce qui l'inspire et la soutient désormais, c'est la conviction que, eu égard à tous les aspects de la situation, il doit satisfaire la volonté exprimée par le patient dans cette demande, volonté avec laquelle lui, le médecin, peut en définitive être en accord. Sur quoi repose cet accord, de prime abord problématique ? Sur la «sollicitude critique» évoquée plus haut. Dans la situation envisagée ici, une telle sollicitude est celle qui, sans dérives, est capable de faire accéder le médecin à une conception de la fidélité envers soi et ses engagements qui le hisse au-delà de sa conception spontanée. Elle place au premier plan, non ce qu'il avait posé d'abord comme norme, mais le patient reconnu, lui aussi, comme un être d'initiative et de choix. Lorsque le médecin accomplit cette démarche dans un parcours intérieur exigeant, l'intégrité $\mathrm{du}$ patient et la sienne acquièrent une portée nouvelle. Pour le médecin, il ne s'agit plus seulement de respecter l'intégrité physique, même entendue au sens du devenir singulier de l'être biologique, mais aussi l'intégrité morale de l'un et de l'autre comprise comme affirmation authentique de soi.

\section{***}

Revenons aux trois lois belges adoptées en 2002. Chacune exerce une influence considérable. La loi sur les droits du patient est aujourd'hui toujours en cours d'acclimatation. En effet, elle bouleverse les pratiques inspirées de toutes les formes de paternalisme médical, et il serait abusif

\section{POUR BON NOMBRE DE CEUX -}

PATIENTS, MÉDECINS

OU AUTRES - QUI SONT

CONFRONTÉS, PAR LA

PRATIQUE OU LA RÉFLEXION,

AUX QUESTIONS QUE SOULÈVE

LA FIN DE VIE, LA POSSIBILITÉ

DE PRATIQUER L'EUTHANASIE

A TRANSFORMÉ LE RAPPORT

À LA MORT : CELLE-CI EST

DEVENUE NON SEULEMENT

UNE ÉTAPE DE LA VIE

QU'IL IMPORTE DE BIEN TRAVERSER,

MAIS AUSSI UNE ÉTAPE

DONT ON PEUT PARLER PLUS

SEREINEMENT, AVEC LAQUELLE

\section{ON PEUT APPRENDRE À VIVRE.}

de considérer que celles-ci ont totalement disparu. Cependant, cette loi constitue une référence d'autant plus décisive qu'elle est, comme toute loi, contraignante. Elle impose donc une nouvelle manière de concevoir la relation médecin-patient, que les médecins apprivoisent pas à pas.

Du fait de leur adoption presque simultanée, la loi relative à l'euthanasie et la loi relative aux soins palliatifs montrent la volonté du législateur d'offrir ces deux possibilités sur le territoire belge. Les médecins belges dont on peut lire ici les réflexions ${ }^{6}$ récusent tous la polarisation souvent établie entre soins palliatifs et euthanasie, et soulignent au contraire la complémentarité entre ces deux approches.

Ces deux lois ont aussi contribué, chacune à leur manière, à transformer la conception de la fin de vie. Dans le cadre des soins palliatifs, la fin de vie peut constituer une tranche de vie au cours de laquelle le soulagement de la douleur et la recherche de la meilleure qualité possible de vie dans tous ses aspects seront des objectifs prioritaires. Quant à la possibilité de demander l'euthanasie, elle permet de ne plus voir la mort comme la grande faucheuse qui vous prend par surprise. La mort suscite toujours l'effroi et la peur. Mais le fait de pouvoir en décider le moment contribue à l'apprivoiser. Pour bon nombre de ceux - patients, médecins ou autres - qui sont confrontés, par la pratique ou la réflexion, aux questions que soulève la fin de vie, la possibilité de pratiquer l'euthanasie a transformé le rapport à la mort: celle-ci est devenue non seulement une étape de la vie qu'il importe de bien traverser, mais aussi une étape dont on peut parler plus sereinement, avec laquelle on peut apprendre à vivre. La loi relative à l'euthanasie contribue en ce sens à «désensauvager» la mort, ce que, dès le milieu des années 1970, Philippe Ariès et bien d'autres réclamaient et espéraient, pour nos sociétés (Ariès, 1977; Morin, 1976).

Il n'en reste pas moins que la possibilité de pratiquer l'euthanasie confronte à une question morale extrêmement complexe. En effet, elle met en évidence l'opacité de chacun face aux autres: autrui reste toujours pour partie un mystère, même pour ceux qui le connaissent intimement. Le médecin, comme les proches, ne peut que faire confiance à la parole du malade qui, malgré sa peur de la mort, réclame celle-ci au nom d'une conviction intérieure forte et librement forgée. Le respect se hausse alors au-dessus du respect de la vie physique pour devenir le respect de ce mystère insondable qu'est l'autre, lui aussi constitutif de son intégrité. Sous conditions, la loi donne à cette exigence morale une force contraignante encore plus nette puisqu'elle en fait une norme sociale. 


\section{Bibliographie}

ARIÈS, PH. (1977). L'homme devant la mort, Paris, Seuil.

BERNARD, CL. (s.d.). Principes de médecine expérimentale, Paris, Masson.

CANGUILHEM, G. (1985). La connaissance de la vie, Paris, Vrin (1 ${ }^{\text {re }}$ éd. 1946).

CHÂTELET, N. (2005). La dernière leçon, $n^{\circ} 1380$, Paris, Seuil (1 ${ }^{\text {re }}$ édition 2004), coll. «Points».

COMITÉ CONSULTATIF DE BIOÉTHIQUE DE BELGIQUE, Avis $n^{\circ} 1$ du 12 mai 1997 concernant l'opportunité d'un règlement légal de l'euthanasie.

COMITÉ CONSULTATIF DE BIOÉTHIQUE DE BELGIQUE, Avis $n^{\circ} 9$ du 22 février 1999 concernant l'arrêt actif de la vie des personnes incapables d'exprimer leur volonté.

DUPONT, B.-M. (2011). D'un prétendu droit de mourir par humanité. L'euthanasie en question, Paris, François Bourin Éditeur.

EY, H. (1981). Naissance de la médecine, Paris, Masson.

GAUCHET, M. (1998). La religion dans la démocratie. Parcours de la laïcité, Paris, Gallimard.

GAUCHET, M. (2002). "Les tâches de la philosophie politique», dans Revue du Mauss, n' 19 , p. 275-303.

HERMEREN, G. (2001). "Intégrité», dans Hottois G. et J.-N. Missa (dir.), Nouvelle encyclopédie de bioéthique. Médecine, environnement, biotechnologie, Bruxelles, De Boeck Université, p. 542-544.

HERREMANS, J. (2011/2012). "Mourir dans la dignité. La loi belge relative à l'euthanasie, une réponse légale ", Frontières, vol. $24, n^{\text {os }} 1-2$, p. 73-80.

LALLEMAND, R. et P. DE LOCHT (2001). L'euthanasie, Bruxelles, EVO.
LOIS BELGES (pour voir les modifications apportées, consulter $<$ http://www.cass.be $>$ ) :

Loi du 28 mai 2002 relative à l'euthanasie, Moniteur belge, 22 juin 2002.

Loi du 14 juin 2002 relative aux soins palliatifs, Moniteur belge, 26 octobre 2002

Loi du 22 août 2002 relative aux droits du patient, Moniteur belge, 26 septembre 2002.

MORIN, E. (1976). L'homme et la mort, Paris, Seuil, coll. «Points».

RICOEUR, P. (1990). Soi-même comme un autre, Paris, Seuil, coll. "L'ordre philosophique».

RICOEUR, P. (2001). "Les trois niveaux du jugement médical », dans Le Juste 2, , Paris, Éd. Esprit, coll. «Philosophie».

SALMON, R. (2007). Tout ce que les chirurgiens ne peuvent pas vous dire, Paris, Anne Carrière.

TAYLOR, CH. (1994). Le malaise de la modernité, Paris, Cerf, coll. «Humanités ».

VAN NESTE, F. (2011/2012). «Travaux de la Commission fédérale belge de contrôle et d'évaluation de l'application de la loi du 28 mai 2002 relative à l'euthanasie », Frontières, vol. 24 , nos $1-2$, p. $81-87$.

\section{Notes}

1. Pour une approche détaillée de ces conditions, voir dans ce numéro : J. Herremans, «Mourir dans la dignité. La loi belge relative à l'euthanasie, une réponse légale», et $\mathrm{F}$. Van Neste, "Travaux de la Commission fédérale belge de contrôle et d'évaluation de l'application de la loi du 28 mai 2002 relative à l'euthanasie », particulièrement la section "Demande volontaire, réfléchie, répétée et sans pression extérieure ", in fine et la note 9 ainsi que la section "Traitements médicaux en fin de vie et euthanasie».
2. Aux côtés de Roger Lallemand, Pierre de Locht s'est fortement engagé dans les débats éthico-législatifs de la société belge, tant l'interruption volontaire de grossesse que l'euthanasie, en prenant distance par rapport aux positions officielles de l'Église catholique de Belgique.

3. Sur les responsabilités du médecin, voir dans ce numéro l'article de J. Herremans et celui de F. Van Neste: section «Le travail d'évaluation de la commission».

4. Voir en ce sens : le concept d' «idiosyncrasie» déjà mis en évidence par Claude Bernard, Principes de médecine expérimentale, Paris, Masson (s.d.), p. 219-221, ainsi que par Georges Canguilhem (1985), La connaissance de la vie, Paris, Vrin (1 ${ }^{\text {re }}$ éd. 1946), p. 146: "le vivant apporte ses propres normes d'appréciation des situations, où il domine le milieu, et se l'accommode». Voir aussi Bertrand-Marie Dupont (2011), D’un prétendu droit de mourir par humanité. L'euthanasie en question, Paris, François Bourin Editeur, p. 21-26 qui montre les prolongements actuels de cette tension entre singulier et connaissance objective.

5. Dans un tout autre mode d'expression, on retrouve les aspects mis en évidence par $\mathrm{H}$. Ey dans le témoignage du $\mathrm{D}^{\mathrm{r}}$ Rémy Salmon (2007), Tout ce que les chirurgiens ne peuvent pas vous dire, Paris, Anne Carrière.

6. Voir dans ce numéro les articles des médecins belges Philippe Van Vlaenderen, Gérard Marin et Nadia Huart. 